\title{
Necroptosis-related Prognostic Gene Signature to Aid Therapy in Lung Adenocarcinoma
}

\section{Yuqi Song}

First Hospital of Jilin University

Jinming Zhang

Second Affiliated Hospital of Jilin University

Linan Fang ( $\nabla$ fanglinan_jdyy@jlu.edu.cn )

First Hospital of Jilin University

Wei Liu

First Hospital of Jilin University

\section{Research Article}

Keywords: Lung adenocarcinoma, necroptosis, gene signature, prognostic, immunotherapy, chemotherapy

Posted Date: February 18th, 2022

DOI: https://doi.org/10.21203/rs.3.rs-1331421/v1

License: (c) (1) This work is licensed under a Creative Commons Attribution 4.0 International License. Read Full License 


\section{Abstract}

Lung adenocarcinoma (LUAD) is the most common type of non-small cell lung cancer (NSCLC), which is also the most important cause of cancer-related deaths. Necroptosis is a phenomenon of cellular necrosis resulting from cell membrane rupture by the corresponding activation of Receptor Interacting Protein Kinase 3 (RIPK3) and Mixed Lineage Kinase domain-Like protein (MLKL) under programmed regulation. It is reported that necroptosis is closely related to the development of tumor, but the prognostic role and biological functions of necroptosis in LUAD are still obscure. In this study, we constructed a prognostic Necroptosis-related gene (NRG) signature based on the RNA transcription data of LUAD patients from The Cancer Genome Atlas (TCGA) and Gene Expression Omnibus (GEO) databases as well as the corresponding clinical characteristics information. Next, Kaplan-Meier analysis, receiver operating characteristic (ROC), univariate Cox and multivariate Cox regression were made to validate and evaluate the model. We also accurately and quantitatively predicted the prognosis of LUAD patients using our nomogram, which includes age, gender, stage and risk score. To improve patients' prognosis and achieve personalized precision medicine, we analyzed the immune landscape in LUAD and predicted the sensitivity of LUAD patients with different risk scores to immunotherapeutic regimens and chemotherapeutic regimens. In conclusion, our study provides a reference for in-depth mechanistic exploration of necroptosis in LUAD.

\section{Introduction}

As the most important cause of cancer death, lung cancer has been a major research topic for clinicians and researchers ${ }^{1}$. Non-small cell lung cancer (NSCLC), the most important type of lung cancer, accounts for $85 \%$ of the total incidence of the disease ${ }^{2}$. Lung adenocarcinoma (LUAD) is the most common pathological type of NSCLC. It generally grows slowly, has a long multiplication time, and develops insidiously. In addition, lung adenocarcinoma is prone to hematogenous metastasis, so some patients are often diagnosed at advanced stages of adenocarcinoma, and their clinical outcome is poor ${ }^{3}$. In recent years, with the development of RNA sequencing, microarrays and other "Omics" technologies, a series of new potential markers driving tumor cell formation have been identified and progressively applied in the clinic. The average 5-year survival rate of LUAD patients, although significantly improved, is still less than optimal and the patients' overall survival rate remains low.

Necroptosis was first discovered in 2005 by Degterev A et al. It is a phenomenon of cellular necrosis resulting from cell membrane rupture by the corresponding activation of Receptor Interacting Protein Kinase 3 (RIPK3) and Mixed Lineage Kinase domain-Like protein (MLKL) under programmed regulation ${ }^{4}$. Necroptosis has a proper regulatory mechanism. With the advancement of basic research, necroptosis has been found to be not only involved in the inflammatory pathological mechanism of the body ${ }^{5}$, but also closely related to the development of tumor and the mechanism of drug resistance. On the one hand, inducing necroptosis can remove chemotherapy-resistant tumor cells; on the other hand, while necroptosis exerts anti-tumor effects, it may also kill normal cells and lead to inflammatory responses, 
thus promoting tumor progression and metastasis ${ }^{6}$. In addition, recent studies have shown that induction of necroptosis can act synergistically with immune checkpoint inhibitors (ICls) to enhance the antitumor activity of ICls in drug-resistant tumors ${ }^{7}$. However, the mechanism of the role of necroptosis in LUAD is still unclear, and its relationship with the treatment and prognosis of LUAD patients has been little studied.

In our present study, we downloaded RNA expression profiles of LUAD patients from The Cancer Genome Atlas (TCGA) and Gene Expression Omnibus (GEO) databases. From previous reviews we learned and downloaded 17 genes associated with necroptosis. A prognostic signature containing 5 key NecroptosisRelated Genes (NRGs) was then constructed by a series of bioinformatics algorithms. Based on the expression level of NRGs in LUAD patients, we classified patients into high- and low-risk subgroups. We then performed subgroup analyses to validate the robustness of the signature, analyzed the prognostic variability between high- and low-risk subgroups, explored the functional pathways and signaling pathways involved in key genes, and further investigated the relationship between risk scores and immune cell infiltration, tumor stem cell index, and drug sensitivity.

\section{Results}

\section{Expression and Mutation of Necroptosis-Related Genes in Lung Adenocarcinoma}

We obtained 17 Necroptosis-Related Genes (RIPK1, RIPK3, MLKL, TLR2, TLR3, TLR4, TNFRSF1A, PGAM5, ZBP1, NR2C2, HMGB1, CXCL1, USP22, TRAF2, ALDH2, EZH2, NDRG2) from previous literature reviews. IHC staining results provided levels of 9 of the 17 necroptotic proteins between LUAD and normal lung tissues (Supplementary Figure S1). Fig.1A demonstrated the mutation of NRG in lung adenocarcinoma. Gene mutations occurred in $17.29 \%$ of LUAD samples, of which TLR4, TLR2, EZH2, RIPK1, and NDRG2 were the five genes with the highest mutation frequency. The mutation frequency of TLR4 accounted for $10 \%$, which was significantly higher than the other genes. The different colors in the legend below the image represent different types of genetic mutations. As can be seen from the graph, the most frequent type of mutation is MISSENSE MUTATION.

In the CNV analysis we found that the copy number of necroptosis genes were both amplified and deleted. Among them, ZBP1, RIPK1, and TRAF2 genes were significantly amplified, while HMGB1, TLR3, and ALDH2 were very significantly deleted (Fig.1B). Fig.1C showed the position of the genes on the chromosome. The red nodes represent gene amplification. The blue nodes represent gene deletion.

\section{Identification and Validation of NRG Prognostic Signature}

We randomized 902 patients included in the study into the Train and Test groups. A total of 11 NRGs significantly associated with prognosis were obtained by univariate Cox regression analysis between the expression of NRG and patients' overall survival. After that, based on the prognosis-related NRG expression, LASSO regression analysis was performed on the Train group samples to construct a prognostic signature that included five NRGs (Fig.2A-B). These five genes and their correlation 
coefficients in the signature were shown in Table 1. Then we divided the entire sample into low-risk and high-risk subgroups based on the median risk score, and survival curves were plotted using Kaplan-Meier analysis. The curves showed that the OS of the high-risk subgroup is much lower than that of the low-risk subgroup in both the Train and Test groups (Fig.2C-E\p『0.001).

To assess the prognostic predictive performance of the signature, we plotted ROC curves (Fig.2F-H) and the AUC values in the Test group reached $0.763,0.717$, and 0.729 at year 1,2 , and 3 , respectively, demonstrating the good performance of the signature in assessing prognosis.

Principal components analysis (Fig.2I-K) showed a significant bivariate distribution of patients in these two subgroups.

\section{Risk Score Has Independent Prognostic Significance}

Fig.3A demonstrated the relationship between risk score, patient survival and gene expression of necroptosis regulator. The heat map showed that RIPK3, TLR2 and ALDH2 were lowly expressed in the high-risk subgroup, which corresponds to their correlation coefficients in the predictive signature(Table.1).

We performed univariate and multivariate Cox analyses to test whether the 5-gene signature was an independent predictor of OS in patients with LUAD. Univariate Cox regression analysis(Fig.3B) showed a significant association between risk score and OS. (Train group: HR 2.089, 95\% confidence interval [Cl] 1.657-2.633, $\mathrm{p}<0.001$; Test group: HR 6.481, 95\% Cl 3.095-13.572, $\mathrm{p}<0.001)$. After adjusting for other confounding variables, the five-gene signature remained an independent indicator of OS in multivariate Cox regression studies (Fig.3C) (Train group: HR 2.048, 95\% Cl 1.583-2.650, $p<0.001$; Test group: HR $5.643,95 \% \mathrm{Cl} 2.495-12.7643, \mathrm{p}<0.001)$.

The Sankey diagram (Fig.3D) illustrated the correspondence between the risk score, pathological stage, gender and survival status in LUAD patients.

\section{Nomogram Could Predict Patients' Prognosis}

We developed a nomogram containing risk scores to quantitatively predict the prognosis of LUAD patients (Fig.4A). Gender, age and tumor stages were also included in the nomogram. The AUCs of patients at years 1,2 and 3 were $0.729,0.727$ and 0.736 , respectively (Fig.4B). Combined with the calibration curves of the nomogram shown in Fig.4C, the results show that the nomogram model has very good predictive performance for prognosis.

\section{GO Analysis and KEGG Analysis}

To explore the preliminary function of the signature composed of these 5 genes, we did GO functional analysis and KEGG pathway enrichment analysis using ClusterProfiler $R$ package (adjusted $p<0.05$, $|\log F C|>1)$. GO analysis showed significant enrichment of genes in programmed necrotic cell death, necrotic cell death, I-kappaB kinase/NF-kappaB signaling, regulation of DNA-binding transcription factor 
activity and other functions (Fig.5A). In KEGG pathway analysis, we learned that these genes are mainly concentrated in Necroptosis, Salmonella infection and TNF signaling pathway (Fig.5B).

\section{Immune Landscape Analysis}

We analyzed the correlation between risk score and immune cell infiltration in LUAD. The results showed a significant positive correlation between risk score and CD8 T cells, Macrophage M0, activated CD4 memory $T$ cells, etc.; and a significant negative correlation between risk score and resting Dendritic cells, etc. (Fig.6A-C). Notably, risk scores were positively correlated with activated Mast cells and CD4 memory T cells, and negatively correlated with inactivated both types of cells (Fig. 6D-G). For NK cells, risk scores were significantly positively correlated with such cells in either the activated or inactivated state (Fig.6HI).

Fig.7A showed the results of the ESTIMATE analysis, and we can see that the immune score and ESTIMATE score were significantly lower in the high-risk subgroup.

\section{Immunotherapy-related analysis}

We performed a TMB correlation analysis, Fig.7B-C, showing that the Tumor Mutation Burden differed significantly between the two subgroups of high and low risk according to the risk score. Risk score and TMB were positively correlated, $r=0.3$. Considering that patients in high- and low-risk subgroups may respond differently to immunotherapy, we further investigated the response to ICl therapy represented by CTLA4/PD-1 inhibitors in both subgroups by ImmunoPhenoScore (IPS) (Fig.7D-G). Regardless of the CTLA4 and PD-1 status being positive or negative, patients in the high-risk subgroup had lower IPS than those in the low-risk subgroup. This suggests that patients in the high-risk subgroup do not show a significant clinical benefit from anti-CTLA4 and/or anti-PD-1 therapy. Our results, taken together, clearly indicate that our risk score is associated with non-response to immunotherapy and that immunotherapy is not recommended for the high-risk subgroup.

In addition to this, we also conducted a study on the relevance of stem cell therapy. Fig.7H showed a corresponding rise in the stem cell index as the risk score increased, which also implies that the higher the content of latent tumor stem cells in the tumor tissue, the more likely the tumor will recur. Because tumor development is associated with the presence of cancer stem cells (CSCs) within the tumor, which play an important role in metastasis and other malignant phenotypes ${ }^{8}$.

\section{High-risk subgroups are more sensitive to chemotherapy}

Finally, we tested the sensitivity of patients in high- and low-risk subgroups to familiar drugs. The R package pRophetic ${ }^{9,10}$ allows us to calculate IC50s for common chemotherapeutic agents in the cohort, including cisplatin, paclitaxel, doxorubicin, rapamycin, etc. The IC50 values suggested that patients with LUAD in the high-risk subgroup were significantly more sensitive to common chemotherapeutic agents such as cisplatin, paclitaxel, docetaxel, doxorubicin and rapamycin (Fig. 8A-E). In contrast, the low-risk 
subgroup had higher sensitivity to some EGFR-TKIs (Fig. 8F-H), suggesting that targeted therapy may provide benefit to these patients.

\section{Discussion}

Lung cancer is the leading cause of death worldwide. Although multimodal treatment strategies, including immunotherapy, radiotherapy and non-invasive surgical resection, have made great progress in recent decades, the outcome of treating lung cancer remains unsatisfactory, with a five-year relative overall survival rate of approximately $18 \%^{11}$. Necroptosis, as a form of programmed cell death, plays an integral role in maintaining the homeostasis of the internal environment and is also involved in the pathological processes of various diseases such as neurodegenerative pathologies, ischemia-reperfusion injury, inflammatory bowel disease, viral infections, and tumor formation. Preliminary studies suggest that necroptosis has a "double-edged sword" role in tumor pathology, which can exert either tumorsuppressive or tumor-promoting effects ${ }^{12-14}$. This close and complex relationship suggests that necroptosis may be an important target for tumor progression and may provide new strategies for tumor prognosis and treatment ${ }^{15}$. However, the specific role of necroptosis regulators in LUAD prognosis and the underlying molecular mechanisms are far from being fully elucidated.

Therefore, in this study, we constructed a prognostic signature associated with NRG based on RNA transcriptome data and corresponding clinical information from 812 patients (Train group) with LUAD randomly selected from the TCGA database and GE072094 dataset, and validated the NRG prognostic signature with relevant data from an additional 90 samples (Test group) in the databases. The KaplanMeier survival curve and the ROC curve together demonstrated the good and accurate prognostic prediction ability of our constructed prognostic signature. Using univariate and multivariate Cox regression analyses within the Train and Test groups, we confirmed that risk scores can be used as independent predictors of prognosis in patients with LUAD, and have a prognostic predictive value for patients with different clinicopathological characteristics.

Smoking has long been recognized as one of the important risk factors for lung cancer ${ }^{16}$. However, according to Jemal A et al, there is an increasing proportion of nonsmoking patients among patients diagnosed with lung cancer, especially among those diagnosed with lung adenocarcinoma ${ }^{17}$. Considering that, we included the patients' smoking history in the Cox regression analysis. The results showed that smoking was not an independent prognostic predictor for patients with LUAD, suggesting an important role of non-smoking risk factors in the development of lung adenocarcinoma. Subsequently, we integrated information on patients' age, gender, tumor stage and risk score to construct nomogram to quantitatively predict patients' 1-, 2- and 3-year survival rates to further facilitate clinical practice. The ROC curves and calibration curves demonstrated the robust efficacy of the predictive nomogram.

A total of five NRGs were included in the prognostic prediction model, which were RIPK3, MLKL, TLR2, TNFRSF1A and ALDH2. Several studies have been conducted to explore the function of these genes, especially the relationship between these genes and necroptosis or cancer. RIPK3 is thought to be a key 
molecular switch for the initiation of necroptosis in cells. In the classical necroptotic pathway, deubiquitinated RIPK1 interacts with RIPK3 via exposure of the RIP homotypic interaction motif (RHIM) structural domain and conformational changes to undergo autophosphorylation and crossphosphorylation with the Fas-associalized protein with death domain (FADD)/caspase 8 together to form amyloid signaling complex necrosis vesicles ${ }^{18}$. In turn, MLKL is recruited and phosphorylated to mediate the execution of necrotic apoptosis. When RIPK1 is deficient, DNA-dependent activator of interferon regulatory factors (DAI), lipopolysaccharide and chemical inducers can directly activate RIPK3 through a non-caspase-dependent mechanism, and activated RIPK3 phosphorylates MLKL to mediate necrosis signaling ${ }^{19}$. It was confirmed that cells underwent necroptosis when RIPK1 was deficient and RIPK3 was overexpressed, and apoptosis occurred when RIPK3 was deficient and RIPK1 was relatively overexpressed ${ }^{20}$. Multiple cancers suppress necroptosis through epigenetic silencing of RIPK3, which is consistent with our obtained finding that the mRNA expression of RIPK3 in the model is negatively correlated with patient risk score.

Unlike RIPK3, although activation of MLKL is the executor of the necroptotic process, the expression of MLKL varies much across cancers, which is related to its complex cytological function. Recent studies have revealed that MLKL has an important role in a variety of non-necroptotic processes such as axonal repair, receptor internalization, extracellular vesicle formation, ligand-receptor degradation, and even in the inhibition of necroptosis ${ }^{21}$. Unfortunately, the exact role of MLKL in cancer progression and metastasis is still unclear.

TLR signaling is triggered by pathogen-associated molecular patterns and induces inflammatory cytokine expression through Myeloid differentiation factor 88 (MyD88)-dependent or non-dependent pathways that activate transcription factors such as NF-KB and IRF3/IRF722. Specifically, TLR2 induces TNF expression mainly through the MyD88-dependent pathway, which can indirectly trigger apoptosis, or triggers the classical pathway of necroptosis through RIP1-RIP3 activation ${ }^{23}$.

Interestingly, while TNF is generally recognized to inhibit or kill tumor cells through multiple links, TNF receptors (TNFR), especially TNFR1, have been found to be upregulated in a variety of tumors, such as ovarian cancer ${ }^{24}$, renal clear cell carcinoma ${ }^{25}$ and acute myeloid leukemia ${ }^{26}$. This may be related to the fact that TNFR1 can mediate cell activation and proliferation signals, induce nitric oxide synthase and interleukin 8 activity, and activate NF-KB pathway ${ }^{27}$. This suggests that TNFR1 may play a role in the accelerated proliferation of cancer cells and altered apoptotic index, which leads to an accelerated cellular metabolic rate and increased chance of mutation. TNFRSF1A, the gene encoding the TNFR1 protein, was similarly found to be highly expressed in high-risk group of LUAD patients in our present study.

ALDH2 belongs to the acetaldehyde dehydrogenase family. Recent studies have found that ALDH2 is not only involved in aldehyde metabolism but also plays a key role in tumor growth. Li et al. found that a decrease in ALDH2 induced not only proliferation and stem cell properties of lung adenocarcinoma cells but also DNA damage and migration while leading to acetaldehyde accumulation. This would promote 
tumor recurrence, drug resistance and metastasis, leading to poor prognosis of lung adenocarcinoma ${ }^{28}$. In addition, chen et al. found that ALDH2 expression was negatively correlated with the expression of DNA base excision repair protein (XRCC1), again implying that low ALDH2 expression would result in lower overall survival ${ }^{29}$.

Tumor immunotherapy is on the rise, and advances in immunotherapy have paved an effective and safe path to rescue patients with NSCLC who lack targeted mutations. However, this approach is also largely influenced by the tumor microenvironment (TME), such as immune cell abundance, tumor mutation burden, and regulatory relationships with other biomolecules ${ }^{30-32}$. We then analyzed the correlation between risk scores and immune cell infiltration in our model. We further did a differential analysis of the relative enrichment of immune cells and stromal cells in LUAD, laying the foundation for subsequent analysis of the feasibility of immunotherapy. Collectively, our findings showed that patients in the highrisk subgroup had lower IPS scores than those in the low-risk subgroup, regardless of CTLA4 and PD-1 status being positive or negative. It clearly indicates that our risk score is associated with non-response to immunotherapy. Therefore, based on the NRG signature, we believe that patients with lower risk scores will be more likely to benefit from immunotherapy.

Biomarkers that predict chemotherapy sensitivity will help physicians select patients more suitable for chemotherapy, which is especially important for patients with lung adenocarcinoma. The robustness of the $\mathrm{R}$ package in predicting response to chemotherapy has been demonstrated in different clinical trials ${ }^{9}$. Using the R package pRRophetic, we found that the high-risk subgroup was significantly more sensitive to most common lung cancer chemotherapies than the low-risk group, such as cisplatin, paclitaxel, doxorubicin, rapamycin, etc. To a certain extent, it compensated for the poor sensitivity of immunotherapy in the high-risk subgroup and provided a new idea to guide the use of chemotherapy as the main treatment modality for LUAD patients.

Of course, our study has many drawbacks. Firstly, necroptosis is a new and rapidly developing field, and more and more necroptosis-related genes will be discovered and fully studied over time. Our findings will be fleshed out then. Secondly, all data samples in this study were obtained from public open-source databases. Due to the relatively small number of LUAD patients in public databases and the duplication of transcriptome data in different databases, the sample size covered in the randomized grouping of this study was relatively insufficient, resulting in low significance of some results. On the other hand, some important clinical details were not available in the open-source dataset, including chemotherapy regimens, drug information and tumor TNM grading. And the lack of these data limits a more in-depth comparison between the TCGA and GEO datasets. Finally, the role of some NRGs in non-small cell lung cancer is unclear and still needs to be revealed by further in vivo or in vitro experiments.

In conclusion, we constructed a robust NRG-related prognostic signature that can be used to predict the prognosis of LUAD patients and develop treatment plans for them. We also accurately predicted the sensitivity of LUAD patients with different risk scores to immunotherapy regimens and chemotherapy 
regimens, which can provide a reference for improving patient prognosis and achieving personalized precision medicine.

\section{Materials And Methods}

\section{Data acquisition}

RNA transcriptome information and clinical information of lung adenocarcinoma patients were obtained from the TCGA database (https://portal.gdc.cancer.gov/) and the GSE72094 dataset in the GEO database (https://www.ncbi.nlm.nih.gov/geo/), respectively. The RNAseq transcriptome data were converted to transcript volume per million (TPM) values, and the R "limma" and "sav" package were applied for batch correction and normalization of RNA-seq from both platforms. After excluding samples with incomplete clinical information or gene expression data, 504 patients from TCGA and 398 patients from GSE72094 dataset with LUAD were included in the downstream analysis. 17 Necroptosis-related Genes (RIPK1, RIPK3, MLKL, TLR2, TLR3, TLR4, TNFRSF1A, PGAM5, ZBP1, NR2C2, HMGB1, CXCL1, USP22, TRAF2, ALDH2, EZH2, NDRG2) were obtained from literature reviews of previous related studies ${ }^{33-41}$. We used the human protein atlas database to demonstrate the proteins encoded by Necroptosis-related Genes.

\section{Gene mutation and gene copy number variation}

The mutations of necroptosis-related genes in TCGA samples were visualized as waterfall plots using the "maftools" package. Copy number variation (CNV) data of LUAD patients were obtained from http://xena.ucsc.edu/. Visualization of the copy number variation frequency of necroptosis-related genes in TCGA samples and demonstration of the relative position of these genes to chromosomes using the $\mathrm{R}$ "RCircos" package.

\section{Construction and validation of a necroptosis-related prognostic signature}

Sample data from the TCGA and GSE72094 dataset was combined, including expression data of necroptosis-related genes and patients' survival data. A univariate Cox regression analysis was performed to obtain gene signature significantly associated with prognosis. After that, we randomly divided the patients into Train and Test groups (812 in the Train group and 90 in the Test group). The R "glmnet" package was used to perform lasso regression analysis on the prognostic data and to optimize the penalty function using cross-validation. A prognostic signature consisting of genes related to necroptosis was developed to predict the prognosis of LUAD patients. The formulae are as follows:

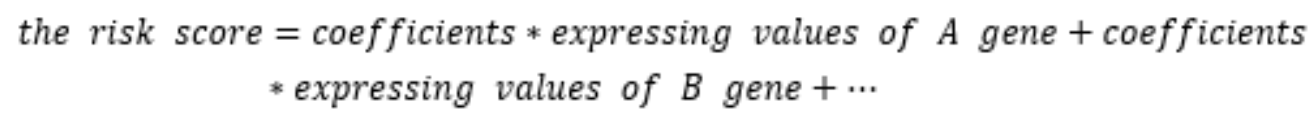

Using the median risk score as the cut-off value, we divided the entire sample into a low-risk subgroup and a high-risk subgroup. Kaplan-Meier analysis was used to plot the overall survival (OS) curves for each group. The R "timeROC" package was used to generate subject operating characteristic (ROC) 
curves, and the area under the curve (AUC) of the ROC curves was measured to show the sensitivity and specificity of the model. Principal component analysis (PCA) was used to examine the distribution of high and low risk subgroups in each group.

In addition, we performed univariate and multivariate Cox regression analyses of the validity of the risk score as an independent prognostic indicator. The clinical characteristics of low- and high-risk patients were compared using the $\mathrm{R}$ "pheatmap" package to explore the correlation between risk scores and clinicopathological variables. Sankey plots were created using the R "ggalluvial" package.

\section{Nomogram construction and verification}

To develop a quantitative prognostic prediction tool for LUAD, we constructed nomogram based on all 902 samples using patient gender, age, pathological stage and risk score as criteria to predict patients' overall survival at 1,2 and 3 years. We then plotted ROC curves and calibration curves to test the validity and robustness of the nomogram.

\section{GO and KEGG analysis}

11 prognosis-related Necroptosis-related Genes were annotated and functionally analyzed using the $\mathrm{R}$ "DOSE" package, including Gene Ontology (GO) and Kyoto Encyclopedia of Genes and Genomes (KEGG), with a corrected $p$-value (q-value) $<0.05$ as the filter.

\section{Correlation between risk score and immune landscape}

To reveal the correlation between risk scores and tumor-infiltrating immune cells, we assessed the immune infiltration of tumors using the CIBERSORT algorithm. We uploaded the full gene expression data of all samples to the CIBERSORTx portal and later ran the algorithm for 1000 permutations based on the LM22 signature. LUAD samples with output $p$-values $<0.05$ were selected for further analysis to explore the relationship between risk score and Necroptosis-related prognostic gene expression and immune cell infiltration. The "estimate" software package was used to calculate the immune score and stromal score for each sample to quantify the relative enrichment of immune and stromal cells in each sample. Violin plots were applied to visualize the differences in enrichment between high- and low-risk subgroups.

\section{Predicting patient response to immunotherapy}

Tumor mutational burden (TMB) is broadly defined as the number of somatic mutations per megabase of interrogated genomic sequence. TMB reflects the total number of mutations carried by tumor cells. It is now generally accepted that TMB is positively correlated with the effect of immunotherapy and can be used as a potential molecular diagnostic marker for tumor immune checkpoint inhibitor therapy ${ }^{42}$. We obtained TMB information for the corresponding TCGA-LUAD cohort from the TCGA database, analyzed the number of somatic nonsynonymous point mutations in each sample using the R "maftools" package. 
In addition, we further explored the potential role of risk scores in the prediction of immunotherapy using the immunophenoscore (IPS). Based on relevant data from The Cancer Imaging Archive (TCIA) database (https://www.cancerimagingarchive.net), we evaluated the differences in four IPS scores between highand low-risk subgroups. The scoring scheme integrates the four major classes of genes that determine tumor immunogenicity (effector cells, immunosuppressive cells, MHC molecules, and immunomodulators) and the gene expression of these cell types (e.g., activated CD4+ T cells, activated CD8+ T cells, effector memory CD4+ T cells, Tregs, MDSCs) to derive specific scores without bias by means of machine learning that is viewed as a new and reliable predictor of response to immunotherapy regimens ${ }^{43}$.

In addition, the relationship between risk score and tumor stemness was evaluated based on the stemness score calculation file obtained from the TCGA database.

\section{Assessment of patients' sensitivity to chemotherapy}

Based on information retrieved from the Genomics of Drug Sensitivity in Cancer (GDSC) database (www.cancerrxgene.org/) and using the R "pRophetic" package, we evaluated the half-inhibitory concentrations (IC50) of different chemotherapeutic agents in each LUAD sample and reflected the differences in chemosensitivity between high- and low-risk subgroups by box plots.

\section{Statistical methods}

The study was statistically analyzed using R programming language (version 4.0.3) and SPSS Statistics 25. The Wilcoxon test was used to analyze continuous variables. Categorical variables were analyzed using Fisher's exact test or chi-square test. Survival differences were analyzed using Kaplan-Meier curves and log-ranch tests. $p$-values $<0.05$ were considered statistically significant.

\section{Declarations}

\section{Data availability}

The datasets analyzed in the current study are available from the TCGA [https://portal.gdc.cancer.gov/], UCSC Xena [http://xena.ucsc.edu/] and GEO [https://www.ncbi.nlm.nih.gov/geo/query/acc.cgi? acc=GSE72094] repository.

\section{Code availability}

The code for generating the risk score model and constructing molecular subtypes discussed in this study is available at Supplementary code.R.

\section{Acknowledgments}

The TCGA and GEO database is gratefully acknowledged for the availability of the raw research data. 
SYQ, ZJM, and FLN made contributions to the study conception and design. SYQ and ZJM collected the clinical information and gene expression data, analyzed the data and wrote the manuscript. SYQ, ZJM, FLN, and LW revised the manuscript. All authors contributed to the article and approved the submitted version.

\section{Funding}

This work was supported by the National Natural Science Foundation of China (\#82003284 to Linan Fang) and the Scientific Research Foundation of The First Hospital of Jilin University (\#JDYY11202015 to Linan Fang).

\section{Additional information}

The authors declare no potential conflicts of interest.

\section{References}

1. Sung, H. et al. Global Cancer Statistics 2020: GLOBOCAN Estimates of Incidence and Mortality Worldwide for 36 Cancers in 185 Countries. CA: A Cancer Journal for Clinicians 71, 209-249, doi:https://doi.org/10.3322/caac.21660 (2021).

2. Chen, Z., Fillmore, C. M., Hammerman, P. S., Kim, C. F. \& Wong, K. K. Non-small-cell lung cancers: a heterogeneous set of diseases. Nat Rev Cancer 14, 535-546, doi:10.1038/nrc3775 (2014).

3. Devarakonda, S., Morgensztern, D. \& Govindan, R. Genomic alterations in lung adenocarcinoma. Lancet Oncol 16, e342-351, doi:10.1016/s1470-2045(15)00077-7 (2015).

4. Degterev, A. et al. Chemical inhibitor of nonapoptotic cell death with therapeutic potential for ischemic brain injury. Nat Chem Biol 1, 112-119, doi:10.1038/nchembio711 (2005).

5. Khoury, M. K., Gupta, K., Franco, S. R. \& Liu, B. Necroptosis in the Pathophysiology of Disease. Am J Pathol 190, 272-285, doi:10.1016/j.ajpath.2019.10.012 (2020).

6. Gong, Y. et al. The role of necroptosis in cancer biology and therapy. Mol Cancer 18, 100, doi:10.1186/s12943-019-1029-8 (2019).

7. Tang, R. et al. Ferroptosis, necroptosis, and pyroptosis in anticancer immunity. J Hematol Oncol 13, 110, doi:10.1186/s13045-020-00946-7 (2020).

8. Wu, J. et al. The long non-coding RNA LncHDAC2 drives the self-renewal of liver cancer stem cells via activation of Hedgehog signaling. J Hepatol 70, 918-929, doi:10.1016/j.jhep.2018.12.015 (2019).

9. Geeleher, P., Cox, N. \& Huang, R. S. pRRophetic: an R package for prediction of clinical chemotherapeutic response from tumor gene expression levels. PLoS One 9, e107468, doi:10.1371/journal.pone.0107468 (2014). 
10. Geeleher, P., Cox, N. J. \& Huang, R. S. Clinical drug response can be predicted using baseline gene expression levels and in vitro drug sensitivity in cell lines. Genome Biol 15, R47, doi:10.1186/gb2014-15-3-r47 (2014).

11. Siegel, R. L., Miller, K. D. \& Jemal, A. Cancer statistics, 2018. CA Cancer J Clin 68, 7-30, doi:10.3322/caac.21442 (2018).

12. Raposo, T. P., Beirão, B. C., Pang, L. Y., Queiroga, F. L. \& Argyle, D. J. Inflammation and cancer: till death tears them apart. Vet J 205, 161-174, doi:10.1016/j.tvjl.2015.04.015 (2015).

13. Liu, X. et al. Key roles of necroptotic factors in promoting tumor growth. Oncotarget 7, 22219-22233, doi:10.18632/oncotarget.7924 (2016).

14. Hänggi, K. et al. RIPK1/RIPK3 promotes vascular permeability to allow tumor cell extravasation independent of its necroptotic function. Cell Death Dis 8, e2588, doi:10.1038/cddis.2017.20 (2017).

15. Philipp, S., Sosna, J. \& Adam, D. Cancer and necroptosis: friend or foe? Cell Mol Life Sci 73, 21832193, doi:10.1007/s00018-016-2193-2 (2016).

16. Gould, M. K. et al. Evaluation of individuals with pulmonary nodules: when is it lung cancer? Diagnosis and management of lung cancer, 3rd ed: American College of Chest Physicians evidencebased clinical practice guidelines. Chest 143, e93S-e120S, doi:10.1378/chest.12-2351 (2013).

17. Jemal, A. et al. Higher Lung Cancer Incidence in Young Women Than Young Men in the United States. N Engl J Med 378, 1999-2009, doi:10.1056/NEJMoa1715907 (2018).

18. Anderton, H. et al. RIPK1 prevents TRADD-driven, but TNFR1 independent, apoptosis during development. Cell Death Differ 26, 877-889, doi:10.1038/s41418-018-0166-8 (2019).

19. Brault, M. \& Oberst, A. Controlled detonation: evolution of necroptosis in pathogen defense. Immunol Cell Biol 95, 131-136, doi:10.1038/icb.2016.117 (2017).

20. Orozco, S. et al. RIPK1 both positively and negatively regulates RIPK3 oligomerization and necroptosis. Cell Death Differ 21, 1511-1521, doi:10.1038/cdd.2014.76 (2014).

21. Martens, S., Bridelance, J., Roelandt, R., Vandenabeele, P. \& Takahashi, N. MLKL in cancer: more than a necroptosis regulator. Cell Death Differ 28, 1757-1772, doi:10.1038/s41418-021-00785-0 (2021).

22. Kumar, H., Kawai, T. \& Akira, S. Pathogen recognition by the innate immune system. Int Rev Immunol 30, 16-34, doi:10.3109/08830185.2010.529976 (2011).

23. Kaiser, W. J. et al. Toll-like receptor 3-mediated necrosis via TRIF, RIP3, and MLKL. J Biol Chem 288, 31268-31279, doi:10.1074/jbc.M113.462341 (2013).

24. Le Page, C. et al. Gene expression profiling of primary cultures of ovarian epithelial cells identifies novel molecular classifiers of ovarian cancer. Br J Cancer 94, 436-445, doi:10.1038/sj.bjc.6602933 (2006).

25. Diegmann, J. et al. Comparative transcriptional and functional profiling of clear cell and papillary renal cell carcinoma. Int J Mol Med 18, 395-403 (2006).

26. Brouwer, R. E. et al. Expression of co-stimulatory and adhesion molecules and chemokine or apoptosis receptors on acute myeloid leukaemia: high CD40 and CD11a expression correlates with 
poor prognosis. Br J Haematol 115, 298-308, doi:10.1046/j.1365-2141.2001.03085.x (2001).

27. Zhou, Z., Connell, M. C. \& MacEwan, D. J. TNFR1-induced NF-kappaB, but not ERK, p38MAPK or JNK activation, mediates TNF-induced ICAM-1 and VCAM-1 expression on endothelial cells. Cell Signal 19, 1238-1248, doi:10.1016/j.cellsig.2006.12.013 (2007).

28. Li, K. et al. ALDH2 Repression Promotes Lung Tumor Progression via Accumulated Acetaldehyde and DNA Damage. Neoplasia 21, 602-614, doi:10.1016/j.neo.2019.03.008 (2019).

29. Chen, X. et al. Interplay between base excision repair protein XRCC1 and ALDH2 predicts overall survival in lung and liver cancer patients. Cell Oncol (Dordr) 41, 527-539, doi:10.1007/s13402-0180390-8 (2018).

30. Herbst, R. S., Morgensztern, D. \& Boshoff, C. The biology and management of non-small cell lung cancer. Nature 553, 446-454, doi:10.1038/nature25183 (2018).

31. Sharma, P., Hu-Lieskovan, S., Wargo, J. A. \& Ribas, A. Primary, Adaptive, and Acquired Resistance to Cancer Immunotherapy. Cell 168, 707-723, doi:10.1016/j.cell.2017.01.017 (2017).

32. He, J., Hu, Y., Hu, M. \& Li, B. Development of PD-1/PD-L1 Pathway in Tumor Immune Microenvironment and Treatment for Non-Small Cell Lung Cancer. Sci Rep 5, 13110, doi:10.1038/srep13110 (2015).

33. Choi, M. E., Price, D. R., Ryter, S. W. \& Choi, A. M. K. Necroptosis: a crucial pathogenic mediator of human disease. JCl Insight 4, doi:10.1172/jci.insight.128834 (2019).

34. Malireddi, R. K. S., Kesavardhana, S. \& Kanneganti, T. D. ZBP1 and TAK1: Master Regulators of NLRP3 Inflammasome/Pyroptosis, Apoptosis, and Necroptosis (PAN-optosis). Front Cell Infect Microbiol 9, 406, doi:10.3389/fcimb.2019.00406 (2019).

35. Xia, X., Lei, L., Wang, S., Hu, J. \& Zhang, G. Necroptosis and its role in infectious diseases. Apoptosis 25, 169-178, doi:10.1007/s10495-019-01589-x (2020).

36. Cheng, M. et al. PGAM5: A crucial role in mitochondrial dynamics and programmed cell death. Eur J Cell Biol 100, 151144, doi:10.1016/j.ejcb.2020.151144 (2021).

37. Wen, S. et al. HMGB1-associated necroptosis and Kupffer cells M1 polarization underlies remote liver injury induced by intestinal ischemia/reperfusion in rats. Faseb j 34, 4384-4402, doi:10.1096/fj.201900817R (2020).

38. Zhu, J. et al. NDRG2 attenuates ischemia-induced astrocyte necroptosis via the repression of RIPK1. Mol Med Rep 22, 3103-3110, doi:10.3892/mmr.2020.11421 (2020).

39. Lou, X. et al. EZH2 Regulates Intestinal Inflammation and Necroptosis Through the JNK Signaling Pathway in Intestinal Epithelial Cells. Dig Dis Sci 64, 3518-3527, doi:10.1007/s10620-019-05705-4 (2019).

40. Petersen, S. L. et al. TRAF2 is a biologically important necroptosis suppressor. Cell Death Differ 22, 1846-1857, doi:10.1038/cdd.2015.35 (2015).

41. Roedig, J. et al. USP22 controls necroptosis by regulating receptor-interacting protein kinase 3 ubiquitination. EMBO Rep 22, e50163, doi:10.15252/embr.202050163 (2021). 
42. Mayakonda, A., Lin, D. C., Assenov, Y., Plass, C. \& Koeffler, H. P. Maftools: efficient and comprehensive analysis of somatic variants in cancer. Genome Res 28, 1747-1756, doi:10.1101/gr.239244.118 (2018).

43. Givechian, K. B. et al. Identification of an immune gene expression signature associated with favorable clinical features in Treg-enriched patient tumor samples. NPJ Genom Med 3, 14, doi:10.1038/s41525-018-0054-7 (2018).

\section{Tables}

Table1 Necroptosis-related Genes and their correlation coefficients in the prognostic signature.

\begin{tabular}{|ll|}
\hline Gene ID & Coefficient \\
\hline RIPK3 & -0.172300828191689 \\
\hline MLKL & 0.168319610189002 \\
\hline TLR2 & -0.153861405082801 \\
\hline TNFRSF1A & 0.40996695235483 \\
\hline ALDH2 & -0.196715171232003 \\
\hline
\end{tabular}

\section{Figures}


A

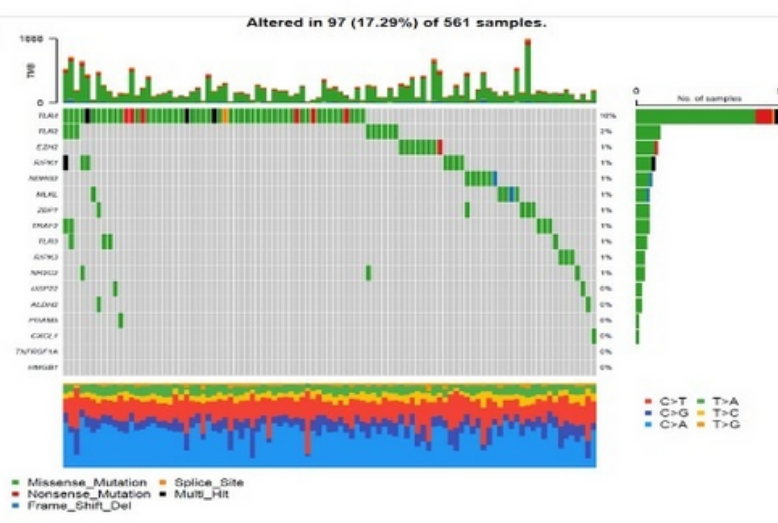

B
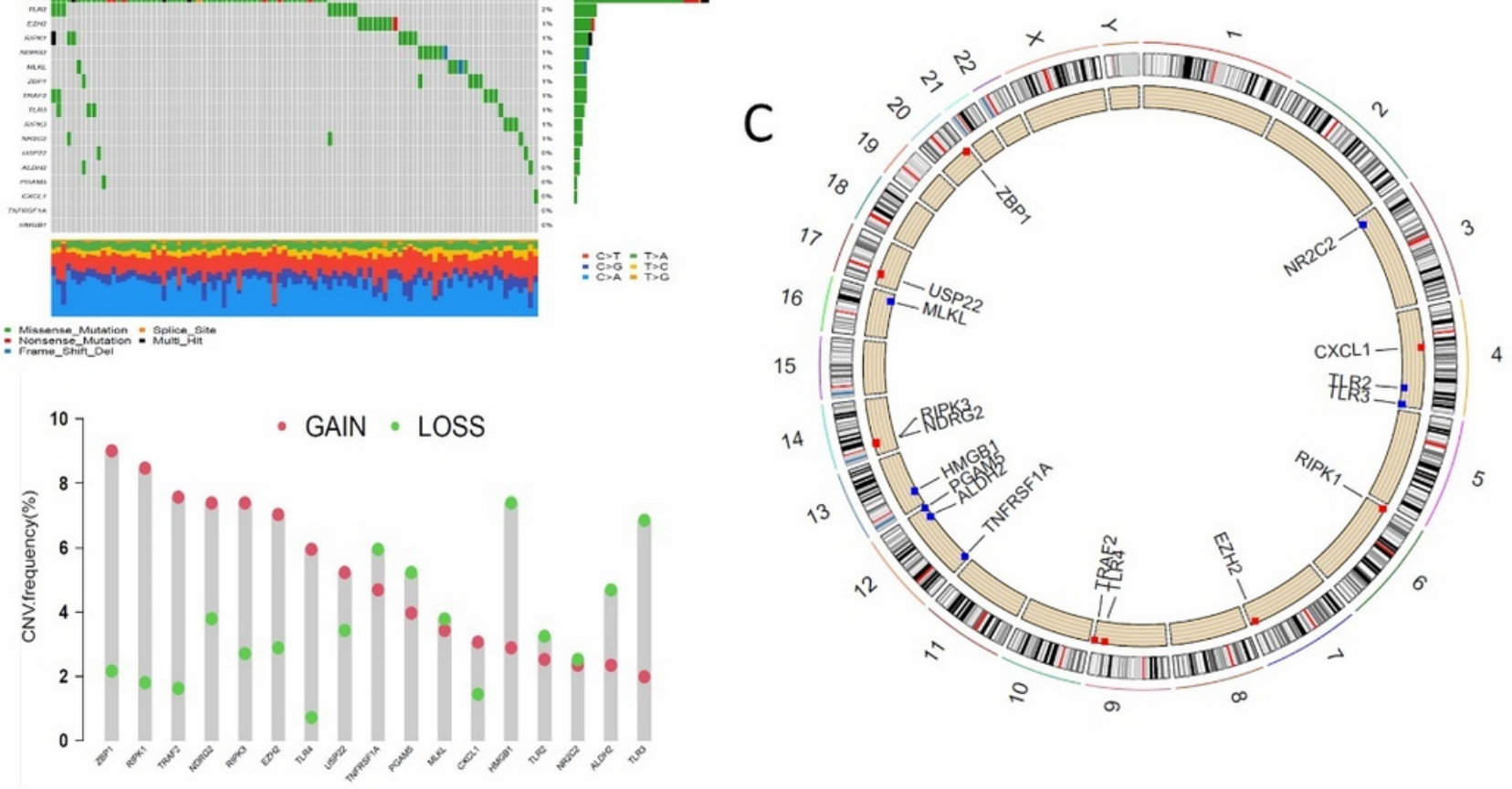

Figure 1

Expression and gene mutation of necroptosis regulators in LUAD. (A) Mutation frequencies of necroptosis regulators in LUAD, different colors represent different types of gene mutations. (B) Copy Number Variation (CNV) frequency of necroptosis genes in LUAD, the height of the column represents the frequency of mutations. (C) Location of Necroptosis-Related Genes on chromosomes. $\left({ }^{*} P<0.05,{ }^{*} P<0.01\right.$, $\star \star \star P<0.001$ ) 

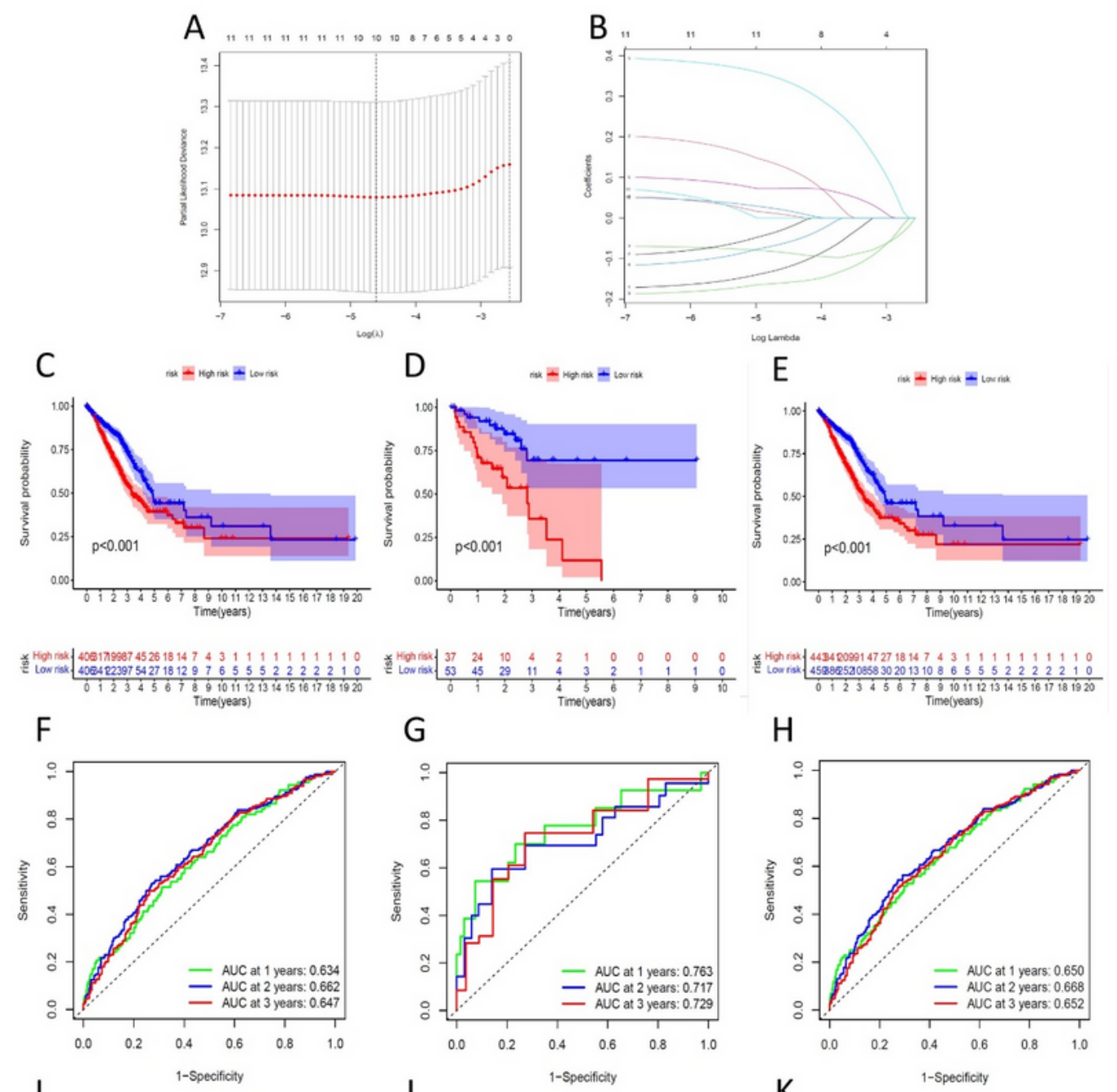

G

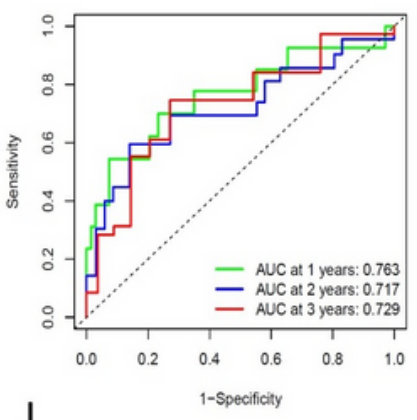

$\mathrm{H}$
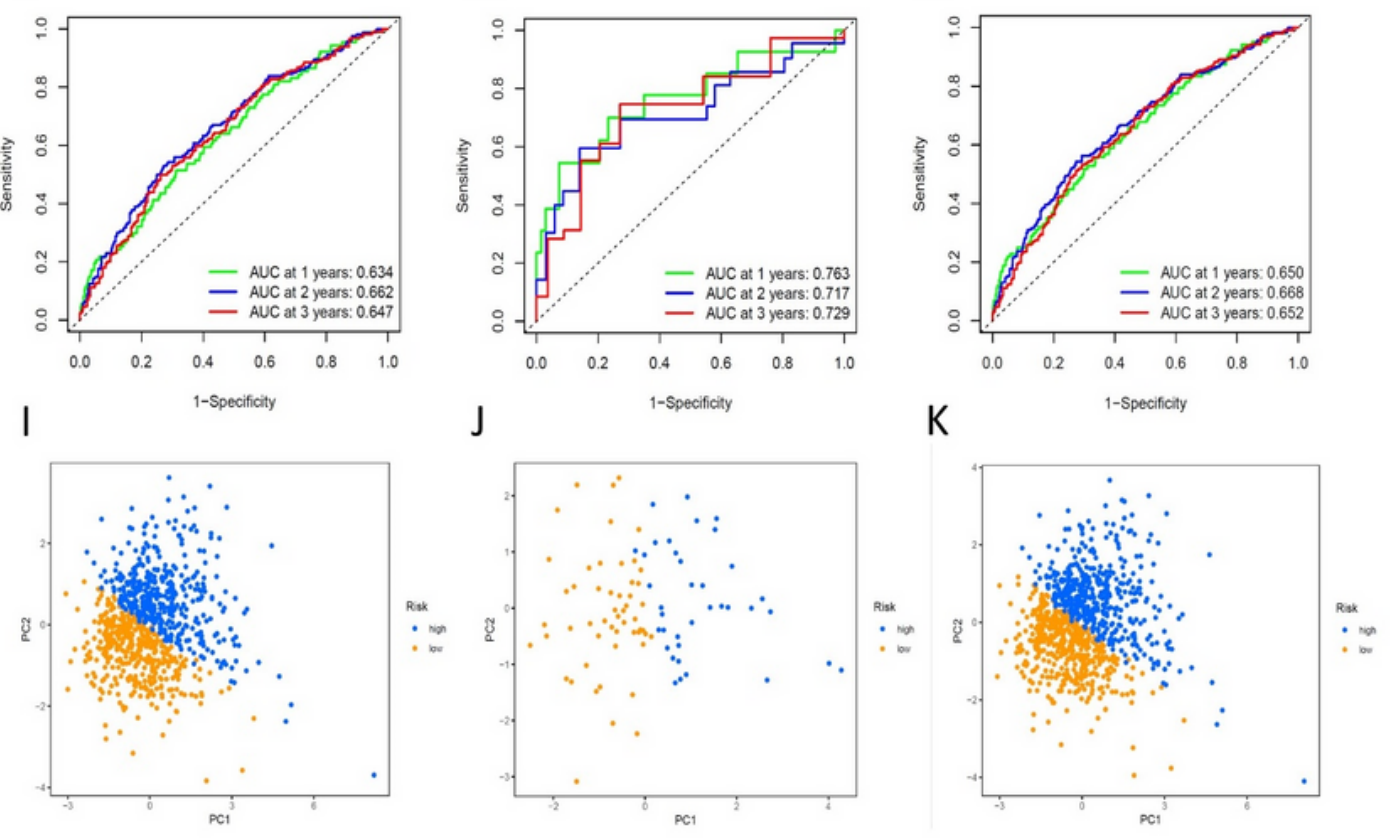

\section{Figure 2}

Establishment and validation of the prognostic signature. (A-B) A LASSO coefficient profiles of 11 candidate genes and an optimal model derived from them. Kaplan-Meier curve analysis presenting difference of overall survival between the high-risk and low-risk subgroups in the Train group (C), the Test group (D), and overall samples (E). 
ROC curves of the NRG signature for predicting the 1/2/3-year survival in the Train group (F), the Test group (G), and overall samples $(\mathbf{H})$.

Principal components analysis in the Train group (I), the Test group (J), and overall samples (K).

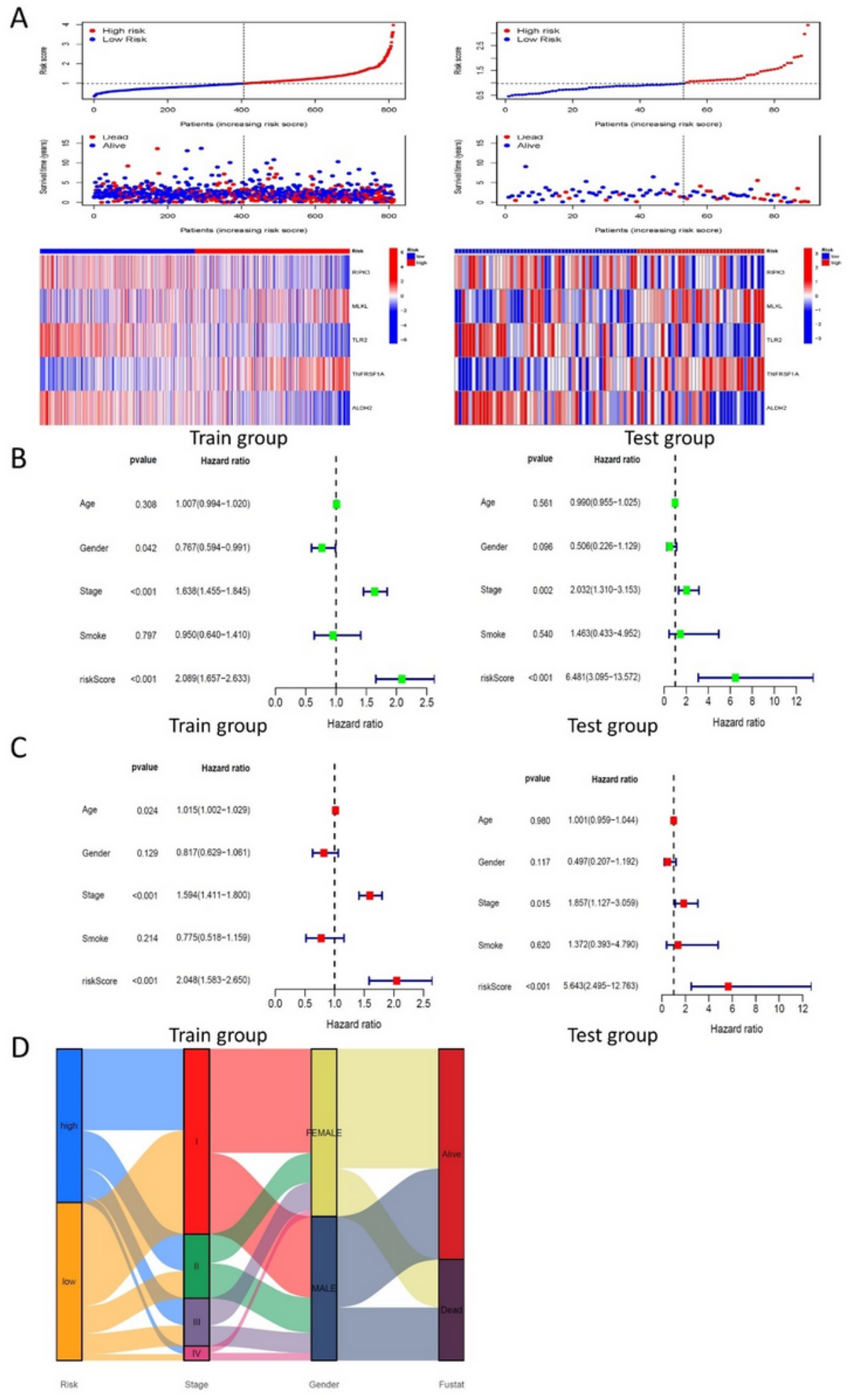

Figure 3 
Risk score has independent prognostic significance. (A) The trends of risk scores, the distribution of survival status and the expression of the 5 genes included in the signature. The prognostic ability and clinical characteristics of the signature were analyzed by univariate Cox regression (B) and multivariate Cox regression (C) in both the Train group and the Test group. (D) The Sankey diagram.

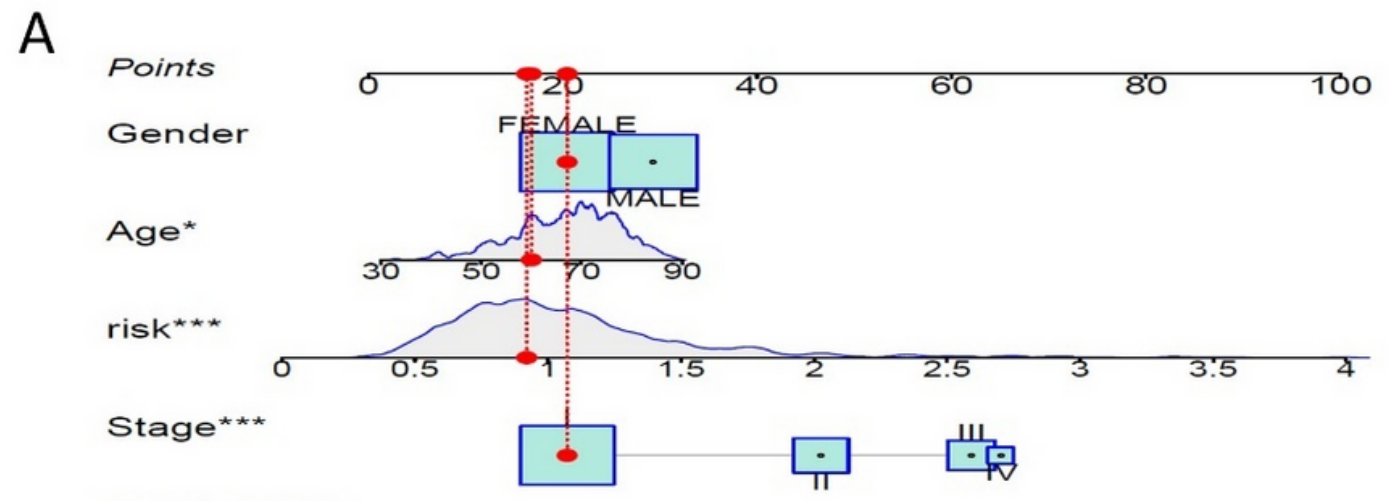

Total points
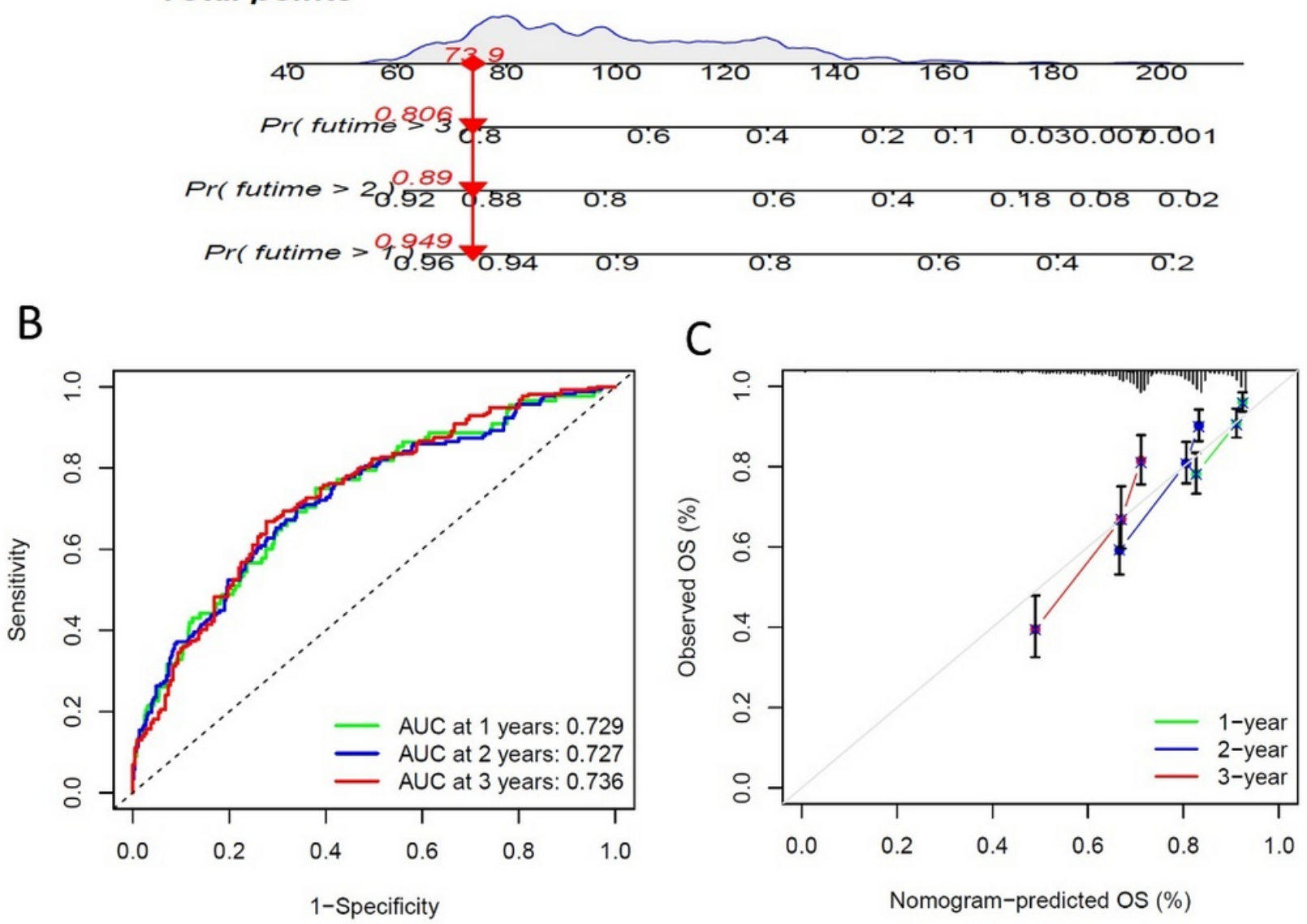

Figure 4

Nomogram was assembled by gender, age, stage and risk score for predicting survival of LUAD patients (A). ROC curves of the nomogram for predicting the 1/2/3-year survival (B). 1/2/3-year nomogram 
calibration curves (C). $\left({ }^{\star} \mathrm{p}<0.05 ;{ }^{\star \star} \mathrm{p}<0.01 ;{ }^{* \star *} \mathrm{p}<0.001\right)$

A

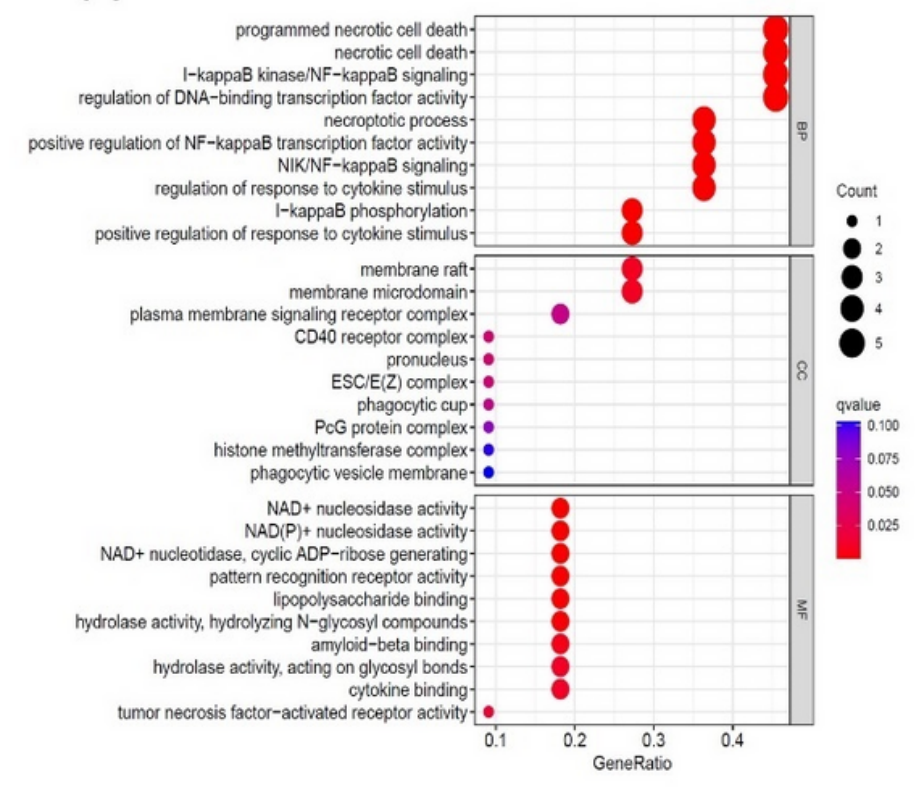

B

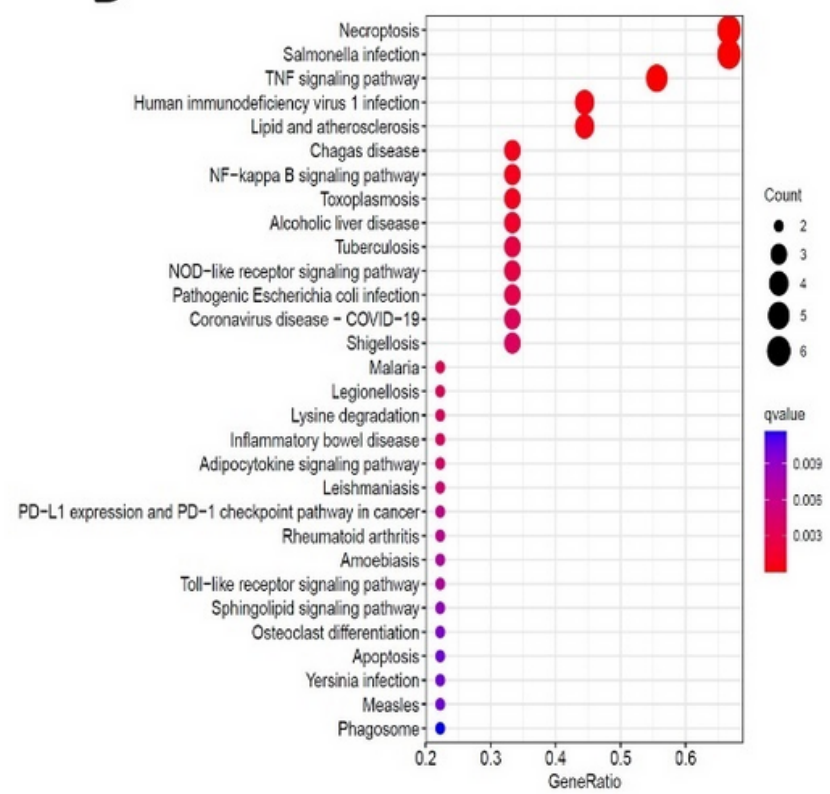

Figure 5

GO enrichment analysis (A) and KEGG enrichment analysis (B) of the 11 prognosis-related Necroptosisrelated Genes. 

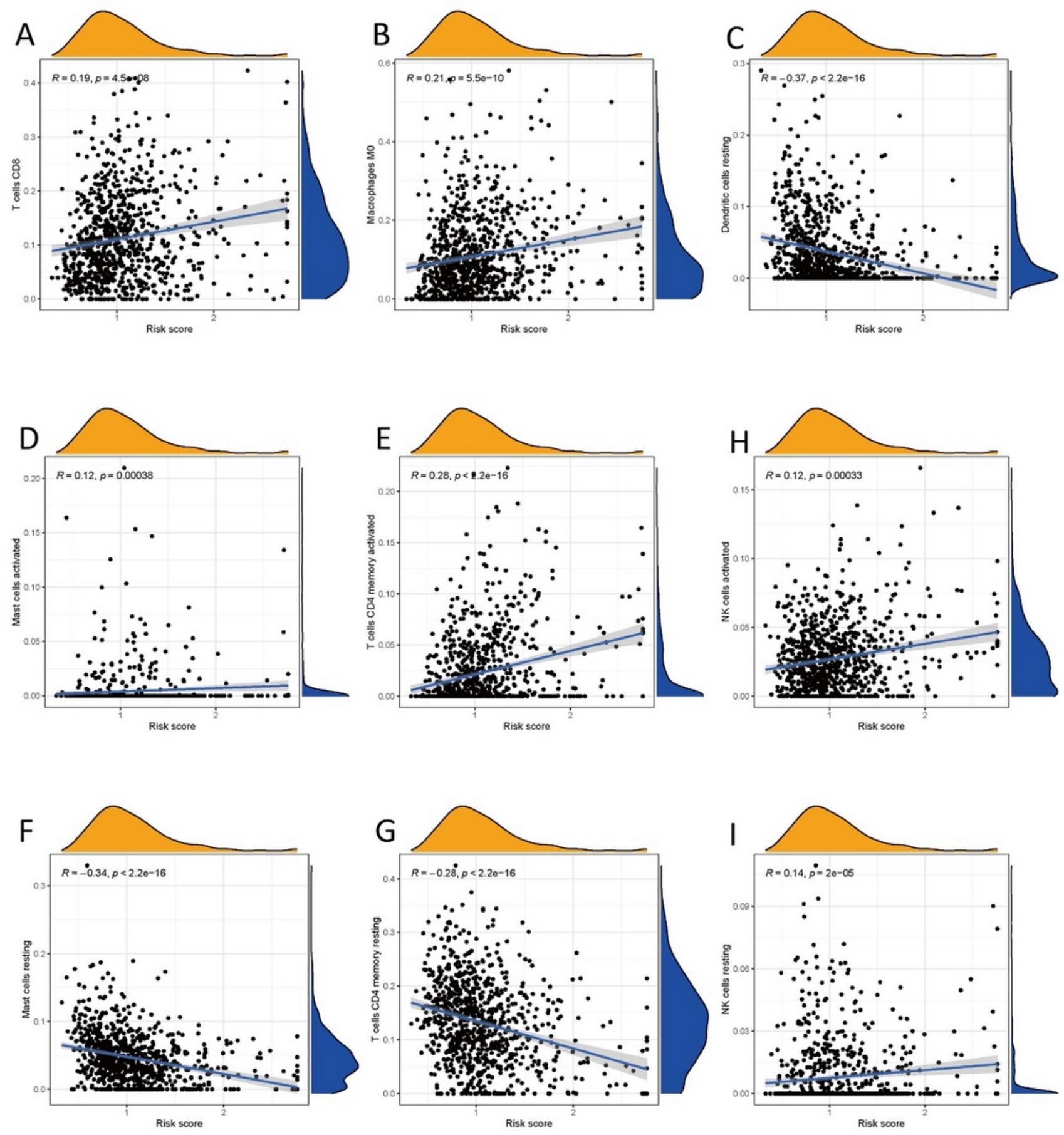

Figure 6

The correlation between risk score and infiltrating immune cells in LUAD. 

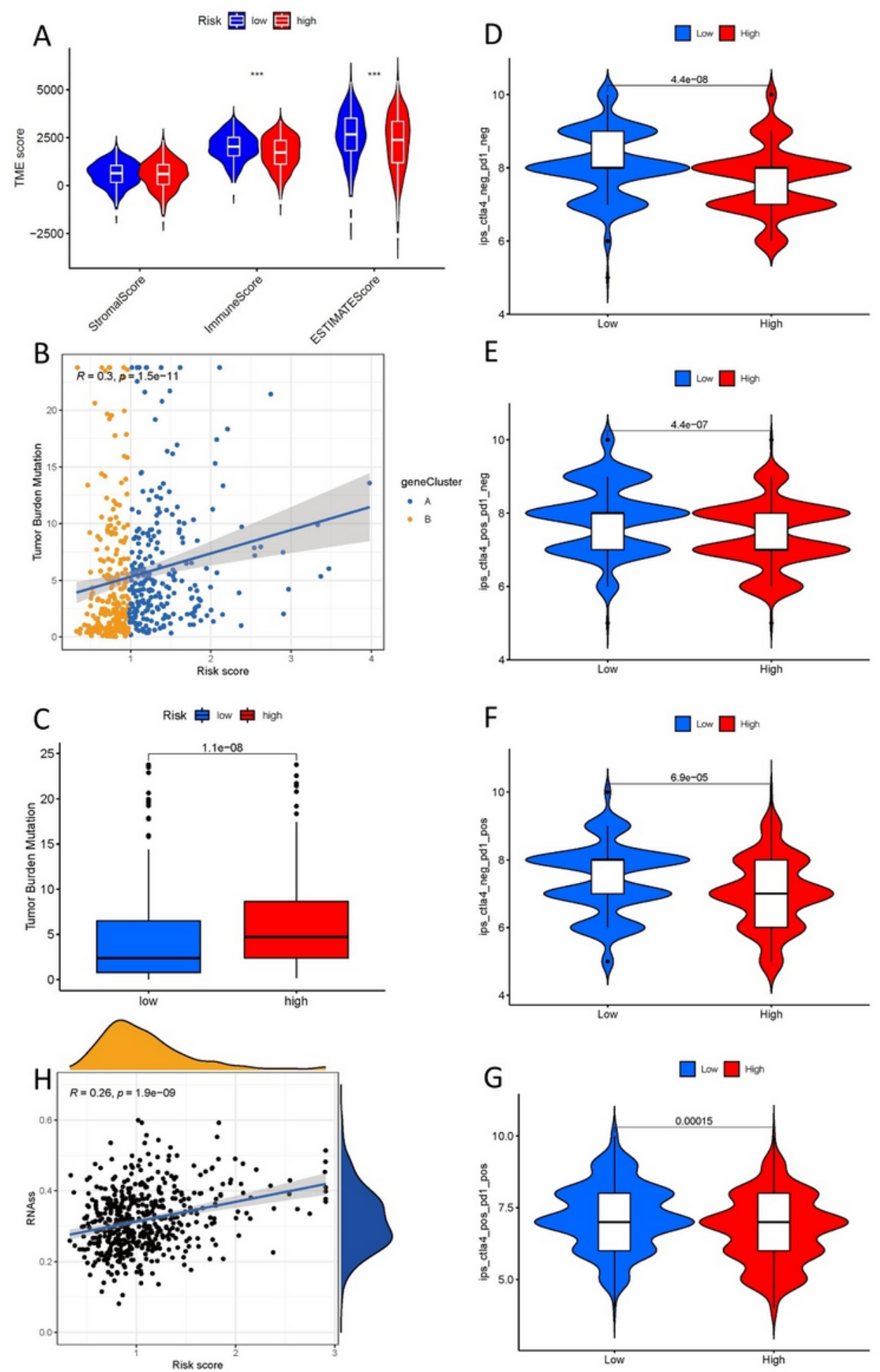

Figure 7

Application of risk score in predicting the immunotherapeutic effect. (A) Differential analysis of tumor microenvironment. Relative enrichment of immune cells and stromal cells. (B-C) Tumor Mutation Burdon Correlation Analysis Showed Positive Correlation Between Risk Score and TMB. The immunophenoscore (IPS) distribution was also compared between high and low risk subgroups (D-G). (H) Stem cell index 
rises with increasing risk score. $\left({ }^{*} p<0.05 ;{ }^{* *} p<0.01\right.$; ${ }^{* \star *} p<0.001$; pos means positive; neg means negative)
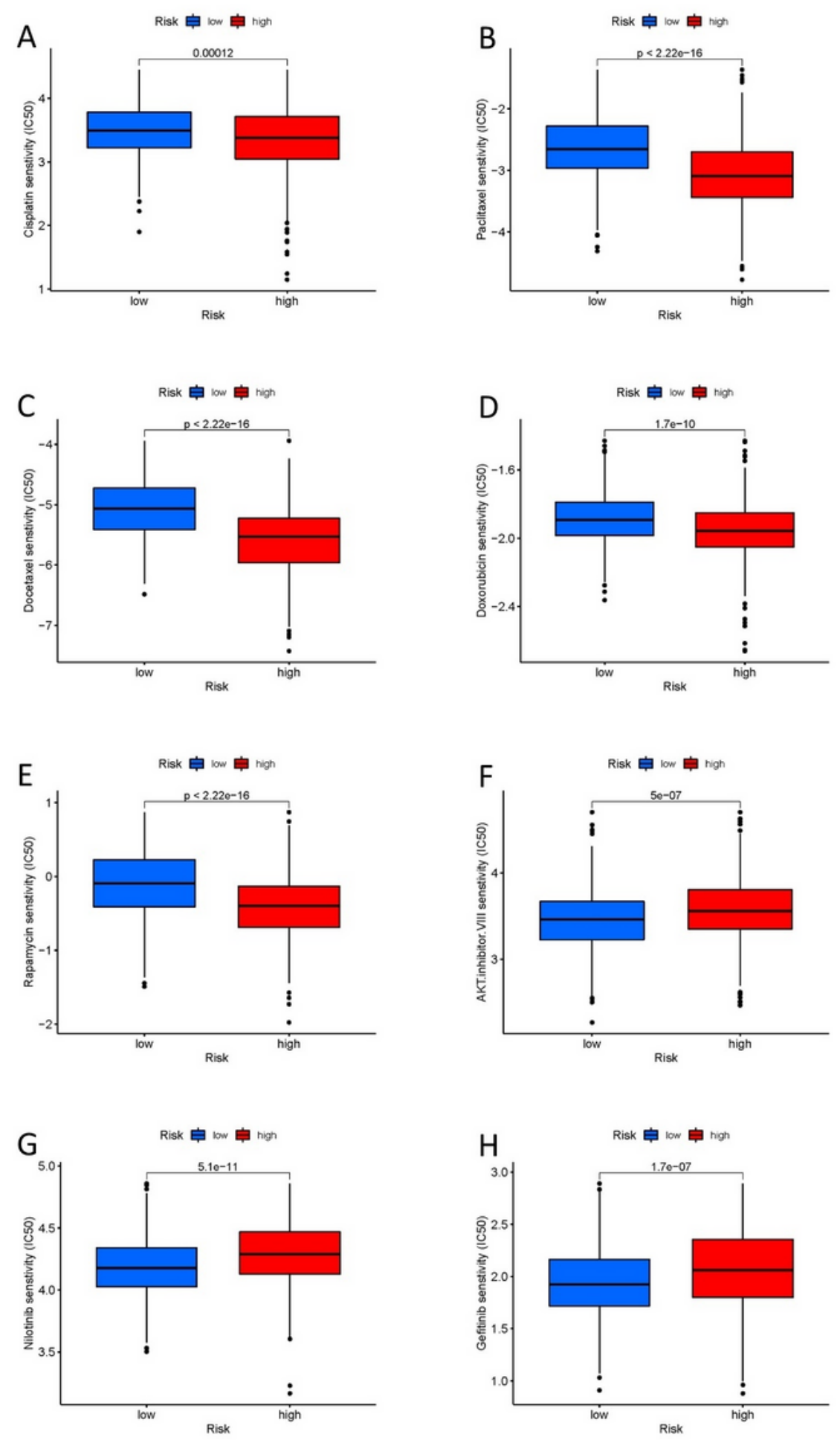

Figure 8

Drug sensitivity of common drugs in high- and low-risk subgroups. 


\section{Supplementary Files}

This is a list of supplementary files associated with this preprint. Click to download.

- SUPPLEMENTARY.pdf

- Supplementarycode.r 